\title{
STRUCTURALLY STABLE GRASSMANN TRANSFORMATIONS
}

\author{
BY
}

STEVE BATTERSON(1)

\begin{abstract}
A Grassmann transformation is a diffeomorphism on a Grassmann manifold which is induced by an $n \times n$ nonsingular matrix. In this paper the structurally stable Grassmann transformations are characterized to be the maps which are induced by matrices whose eigenvalues have distinct moduli. There is exactly one topological conjugacy class of complex structurally stable Grassmann transformations. For the real case the topological classification is determined by the ordering (relative to modulus) of the signs of the eigenvalues of the inducing matrix.
\end{abstract}

1. Introduction and preliminaries. Let $\operatorname{Diff}(M)$ be the space of diffeomorphisms on a compact manifold $M$. Two diffeomorphisms $f, g \in \operatorname{Diff}(M)$ are topologically conjugate, denoted $f \sim g$, if there exists a homeomorphism $h: M \rightarrow M$ such that $h f=g h$. This form of topological equivalence implies that the maps have similar qualitative properties. A diffeomorphism $f$ is structurally stable provided there exists a neighborhood $U$ of $f$ in $\operatorname{Diff}(M)$ such that each $g \in U$ is topologically conjugate to $f$. This means that small perturbations of the function do not alter its topological behavior. Given a manifold $M$ two of the fundamental problems in dynamical systems are:

(1) characterization of the structurally stable maps in $\operatorname{Diff}(M)$,

(2) classification up to topological conjugacy of a large subset of $\operatorname{Diff}(M)$.

In this paper we consider these questions for a subset of the set of diffeomorphisms on a certain class of compact manifolds. Before elaborating on this problem, we will give some basic definitions.

For the following definitions $M$ is a compact manifold, $p \in M$, and $f \in \operatorname{Diff}(M)$. If $f(p)=p$ then $p$ is a fixed point of $f$. If there exists a nonzero integer $m$ such that $f^{m}(p)=p$, then $p$ is said to be a periodic point of $f$. A point $x \in M$ is called a nonwandering point of $f$ provided that for any neighborhood $U$ of $x$ there exists a nonzero integer $m$ such that $f^{m}(U) \cap U \neq \varnothing$. The sets

Received by the editors February 20, 1976.

AMS (MOS) subject classifications (1970). Primary 58F10, 58D05.

Key words and phrases. Grassmann manifold, Grassmann transformation, topological conjugary, structurally stable, Morse-Smale, labelled diagram.

(1) This paper is a portion of the author's doctoral dissertation written under the direction of John Franks at Northwestern University. 
of fixed points, periodic points, and nonwandering points for a diffeomorphism $f$ are denoted respectively by $\operatorname{Fix}(f), \operatorname{Per}(f)$, and $\Omega(f)$. Each of these sets is invariant and is preserved by a topological conjugacy (e.g., $h(\Omega(f))=$ $\left.\Omega\left(h f h^{-1}\right)\right)$.

An invertible linear map on a Banach space is hyperbolic if and only if its spectrum is disjoint from the unit circle. If $p \in \operatorname{Fix}(f)$ then $p$ is a hyperbolic fixed point of $f$ provided the derivative of $f$ at $p, D f(p)$, is a hyperbolic linear map. Suppose $M$ has metric $d$ and $p$ is a hyperbolic fixed point of $f$. The stable manifold of $p, W^{s}(p),=\left\{x \in M \mid d\left(p, f^{m}(x)\right) \rightarrow 0\right.$ as $\left.m \rightarrow \infty\right\}$ and the unstable manifold of $p, W^{u}(p),=\left\{x \in M \mid d\left(p, f^{-m}(x)\right) \rightarrow 0\right.$ as $\left.m \rightarrow \infty\right\}$. These manifolds are also preserved by conjugacies.

A periodic point $p$ of period $m$ is hyperbolic provided that it is a hyperbolic fixed point of $f^{m}$. For a hyperbolic periodic point $p$ the stable and unstable manifolds are defined to be the stable and unstable manifolds of $p$ under $f^{m}$.

A diffeomorphism $f$ on a compact manifold $M$ is said to be Morse-Smale provided it satisfies the following conditions:

(1) $\Omega(f)$ is finite;

(2) all periodic points are hyperbolic;

(3) for each $p, q \in \Omega(f), W^{s}(p)$ and $W^{u}(q)$ have transversal intersection.

The first condition implies $\Omega(f)=\operatorname{Per}(f)$. The following theorem is due to Palis and Smale [10].

Theorem. $f \in \operatorname{Diff}(M)$ is Morse-Smale if and only if $\Omega(f)$ is finite and $f$ is structurally stable.

A hyperbolic structure on $\Omega(f)$ is a continuous splitting of the tangent bundle $T_{\Omega(f)} M$ of $M$ restricted to $\Omega(f), T_{\Omega(f)} M=E^{s} \oplus E^{u}$, invariant under the derivative $D f$, such that $D f$ is contracting on $E^{s}$ and expanding on $E^{u}$. A diffeomorphism $f$ satisfies Smale's Axiom A if and only if $\Omega(f)$ has a hyperbolic structure and $\Omega(f)$ is the closure of $\operatorname{Per}(f)$.

Suppose $f$ satisfies Axiom A. If $x \in \Omega(f)$ then the definitions of stable and unstable manifold can be generalized to

$$
W^{s}(x)=\left\{y \in M \mid d\left(f^{m}(x), f^{m}(y)\right) \rightarrow 0 \text { as } m \rightarrow \infty\right\}
$$

and

$$
W^{u}(x)=\left\{y \in M \mid d\left(f^{-m}(x), f^{-m}(y)\right) \rightarrow 0 \text { as } m \rightarrow \infty\right\} .
$$

If for all $x, y \in \Omega(f), W^{s}(x)$ and $W^{u}(y)$ have transversal intersection then $f$ is said to satisfy the strong transversality condition.

THEOREM. If $f$ satisfies Axiom A and the strong transversality condition then $f$ is structurally stable. 
This theorem was conjectured by Smale and proven by Robbin and Robinson [11], [13]. It is unknown if the converse is true, but hyperbolicity of periodic points is a necessary condition for structural stability [3]. In this paper we will characterize the structurally stable maps in a certain set of diffeomorphisms. The following corollary will simplify this problem:

Corollary. If $f, g, h \in \operatorname{Diff}(M)$ and $h$ is a conjugacy for $f$ and $g$ then

(1) $f$ is Morse-Smale if and only if $g$ is Morse-Smale;

(2) $f$ is structurally stable if and only if $g$ is structurally stable.

Proof of (1). Suppose $f$ is Morse-Smale and $g=h \circ f \circ h^{-1}$. Then $\Omega(g)$ is finite since $\Omega(g)=h(\Omega(f))$. Suppose $g^{m}(p)=p$ and produce $q \in M$ such that $p=h(q)$ and $f^{m}(q)=q$.

Then

$$
\begin{aligned}
T_{p} g^{m} & =T_{p}\left(h \circ f^{m} \circ h^{-1}\right) \\
& =T_{h(q)}\left(h \circ f^{m} \circ h^{-1}\right) \\
& =\left(T_{q} h\right)\left(T_{q} f^{m}\right)\left(T_{q} h\right)^{-1} .
\end{aligned}
$$

Thus $T_{p} g^{m}$ and $T_{q} f^{m}$ have the same eigenvalues and $p$ is a hyperbolic periodic point of $g$.

Suppose $h(p), h(q) \in \Omega(g)$ and $h(x) \in W_{g}^{u}(h(p)) \cap W_{g}^{s}(h(q))$. Then $x$ $\in W_{f}^{u}(p) \cap W_{f}^{s}(q)$.

$$
\begin{aligned}
T_{h(x)} M & =T_{x} h\left(T_{x}(M)\right) \\
& =T_{x} h\left(T_{x} W_{f}^{u}(p)+T_{x} W_{f}^{s}(q)\right) \\
& =\left(T_{x} h\right)\left(T_{x} W_{f}^{u}(p)\right)+\left(T_{x} h\right)\left(T_{x} W_{f}^{s}(q)\right) \\
& =T_{h(x)} h\left(W_{f}^{u}(p)\right)+T_{h(x)} h\left(W_{f}^{s}(q)\right) \\
& =T_{h(x)} W_{g}^{u}(h(p))+T_{h(x)} W_{g}^{s}(h(q)) .
\end{aligned}
$$

Therefore $g$ is Morse-Smale. The proof of part (2) is similar.

Let $G(k, n ; F)$ denote the Grassmann manifold of $k$-dimensional subspaces of $F^{n}$ where $F$ is either $\mathbf{R}$ or $\mathbf{C}$. $\mathrm{Gl}(n ; F)$ will be the general linear group of nonsingular $n \times n$ matrices over $F$. Each $A \in \mathrm{Gl}(n ; F)$ induces a (real or complex) Grassmann transformation $A_{k} \in \operatorname{Diff}(G(k, n ; F))$. Let $\mathscr{T}(k, n ; F)$ be the manifold of Grassmann transformations on $G(k, n ; F)$. Our goals are the following:

(1) Characterize and classify the structurally stable maps in $T(k, n ; F)$.

(2) Analyze an open, dense set in $\mathscr{T}(k, n ; F)$. 
THEOREM A. Let $A \in \mathrm{Gl}(n ; F)$. The following are equivalent:

I. $A_{k}$ is Morse-Smale.

II. $A_{k}$ is structurally stable.

III. The eigenvalues of $A$ have distinct moduli.

COROLLARY. The structurally stable maps form an open, dense, submanifold in $\mathscr{T}(k, n ; \mathbf{C})$ and any two structurally stable maps are topologically conjugate.

The above results (whose proofs are contained in the following section) provide a complete description of the complex case. The structurally stable real Grassmann transformations are neither dense in $\mathscr{T}(k, n ; \mathbf{R})$ nor are they all topologically conjugate to each other. In classifying these maps we use a modification of Smale's definition of labelled diagram. In Theorem $\mathrm{C}$ we obtain a classification based on the ordering (relative to modulus) of the signs of the eigenvalues of the inducing matrix.

Suppose $A, B, P \in \mathrm{Gl}(n ; F)$ and $B=P A P^{-1}$. Then $B_{k}=P_{k} A_{k}\left(P_{k}\right)^{-1}$ and thus the similarity transformation induces a differentiable conjugacy. Consequently in characterizing structurally stable maps and in classifying $\mathcal{T}(k, n ; F)$ it will suffice to consider matrices in the canonical form described below.

An irreducible block is, for some $j \geqslant 1$, a $j \times j$ matrix in one of the following two forms:

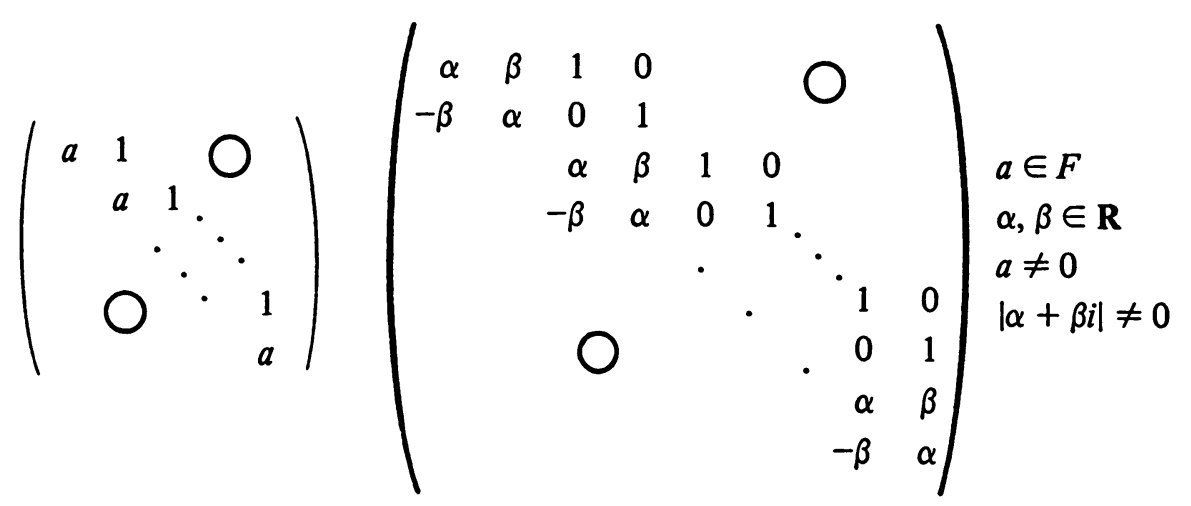

Canonical Form Lemma. If $A \in \mathrm{Gl}(n ; F)$ then $A$ is similar to a matrix of the form

$$
\left(\begin{array}{lll}
\sigma_{1} & & \bigcirc \\
\bigcirc & \ddots & \sigma_{m}
\end{array}\right)
$$

where for each $i$ :

(1) all eigenvalues of $\sigma_{i}$ have the same modulus $\left|\sigma_{i}\right|$,

(2) $\left|\sigma_{i}\right|<\left|\sigma_{i+1}\right|$, 
(3) $\sigma_{i}$ consists of irreducible blocks along its diagonal and 0's elsewhere.

Matrices in this form will be said to be in canonical form.

$\mathrm{N}$. Kuiper has obtained a virtually complete classification of $\mathcal{T}(1, n ; \mathbf{R})[6]$. We state his results in terms of canonical forms.

THEOREM. If two real projective transformations $A_{1}$ and $B_{1}$ can be put in the canonical forms $A=\sigma_{1} \oplus \cdots \oplus \sigma_{m}$ and $B=\tau_{1} \oplus \cdots \oplus \tau_{m}$ and there exist $\lambda_{1}, \ldots, \lambda_{m}>0$ such that, for each $i, \sigma_{i}=\lambda_{i} \tau_{i}$, then $A_{1} \sim B_{1}$.

Kuiper has also proven the converse of this theorem for the transformations in $厅(1, n ; \mathbf{R})$ whose periodic points all have period $q$ or $2 q$ where $q$ is a prime power or 1 .

2. Structurally stable Grassmann transformations. The purpose of this section is to characterize and classify the structurally stable diffeomorphisms in 丁 $(k, n ; F)$.

Definition. Let $c_{\eta_{1}}, \ldots, r_{k}$ be the coordinate plane spanned by the $r_{1}, \ldots, r_{k}$ axes in $F^{n}$. We will assume $r_{i}<r_{i+1}$.

If $A$ is a diagonal matrix, then each $c_{\eta_{1}, \ldots, \eta_{k}}$ is a fixed point of $A_{k}$. We will compute the derivative of $A_{k}$ at $c_{\eta_{1}}, \ldots, \eta_{k}$.

Consider the set of $n \times k$ matrices: $e=e_{\eta_{1}, \ldots, r_{k}}=\left\{\left\{x_{i j}\right\} \mid x_{r_{i} i}=1\right.$ and $x_{r_{i j}}=0$ if $\left.i \neq j\right\}$. The column vectors of each $\left\{x_{i j}\right\}$ span a $k$-dimensional subspace of $F^{n}$. $e$ is a chart for $c_{r_{1}}, \ldots, r_{k}$ with the coordinates of $\left\{x_{i j}\right\}$ being the $k(n-k)$ values of the matrix in the non- $r_{i}$ rows. We will call $\mathcal{C}$ the standard chart for $c_{\eta}, \ldots, \eta_{k}$.

EXAMPLE. $A \stackrel{A}{=} \operatorname{dg}\left(\begin{array}{llll}1 & 2 & 3 & 4\end{array}\right)$,

$$
e_{1,3}=\left\{\left(\begin{array}{cc}
1 & 0 \\
x_{1} & x_{2} \\
0 & 1 \\
x_{3} & x_{4}
\end{array}\right)\right\}
$$

LEMMA. Let $A=\operatorname{dg}\left(a_{1} \cdots a_{n}\right)$ and $c_{r_{1}}, \ldots, r_{k}$ be a coordinate plane in $F^{n}$. Then in $\mathrm{C}$-coordinates the derivative of $A_{k}$ at $c_{r_{1}}, \ldots, r_{k}$ is

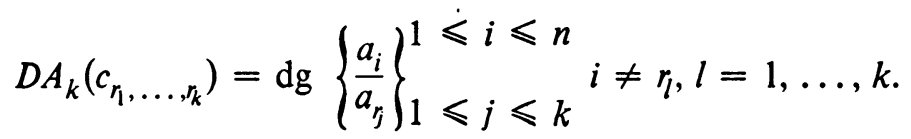

Note. The derivative is a diagonal matrix consisting of all quotients of $\left\{a_{1}, \ldots, a_{n}\right\}$ where the subscript of the denominator element is an $r_{j}$ from $c_{r_{1}, \ldots, r_{k}}$ and the subscript of the numerator is not an $r_{j}$.

EXAMPLE. $A=\operatorname{dg}\left(\begin{array}{llll}1 & 2 & 3 & 4\end{array}\right)$, 


$$
D A_{2}\left(c_{1,3}\right)=\operatorname{dg}(22 / 344 / 3) \text {. }
$$

Proof OF LEMMA.

$$
A\left(\left\{x_{i j}\right\}\right)=\left(\begin{array}{c}
a_{1} x_{1} \\
a_{2} x_{2} \\
\vdots \\
a_{n} x_{n}
\end{array}\right),
$$

$x_{i}$ denotes $i$ th row vector of $\left\{x_{i j}\right\}$. Then $\left(A\left(\left\{x_{i j}\right\}\right)\right)_{r_{i} i}=a_{r_{i}}$ and $\left(A\left(\left\{x_{i j}\right\}\right)\right)_{r_{i j}}=0$ if $i \neq j$.

Let $\left\{y_{i j}\right\}$ be the matrix which results from dividing the $i$ th column vector $(i=1,2, \ldots, k)$ of $A\left(\left\{x_{i j}\right\}\right)$ by $a_{r_{i}}$.

Then $y_{r_{i} i}=1$ and $y_{r_{i} j}=0$ if $i \neq j$. Also $y_{i j}=a_{i} / a_{r_{j}}$ if $i \neq r_{l}, l=1, \ldots, k$. So $\left\{y_{i j}\right\} \in \mathcal{C}$.

Each coordinate $x_{i j}$ of $\left\{x_{i j}\right\}$ is mapped by $A_{k}$ to the coordinate $\left(a_{i} / a_{r_{j}}\right) x_{i j}$. Q.E.D.

In proving the lemma we have also shown that if $A$ is diagonal and $\mathcal{C}$ is a standard chart then $A_{k}(\mathcal{C})=\mathcal{C}$ and $A_{k}$ is linear in standard coordinates on $\bigodot$

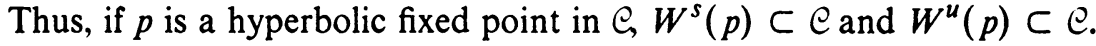

Lemma. If $p \in G(k, n ; F), A=\operatorname{dg}\left(a_{1} \cdots a_{n}\right)$, and $\left|a_{i}\right|<\left|a_{i+1}\right|$ then there exist coordinate $k$-planes $q, q^{\prime} \in G(k, n ; F)$ such that $p$ is forward asymptotic to $q$ and backward asymptotic to $q^{\prime}$.

Proof. Let $p \in G(k, n ; F)$.

If $\vec{v}=\left(v_{1}, \ldots, v_{n}\right) \neq \overrightarrow{0}$, define $s(\vec{v})=\max \left\{j \mid v_{j} \neq 0\right\}$. Choose a basis $\left\{\vec{v}^{i}\right\}_{i=1}^{k}$ for $p$ such that $s\left(\vec{v}^{i}\right)<s\left(\vec{v}^{i+1}\right)$. Then $A^{m}\left(\vec{v}^{i}\right) \rightarrow c_{s\left(\vec{v}^{\prime}\right)}$ as $m \rightarrow \infty$. So $\left(A_{k}\right)^{m}(p) \rightarrow c_{s\left(\vec{v}^{\prime}\right), \ldots, s\left(\vec{v}^{k}\right)}$ as $m \rightarrow \infty$.

Similarly $p$ is backward asymptotic to a coordinate plane.

The following theorem characterizes the structurally stable diffeomorphisms in $\mathcal{T}(k, n ; \mathbf{R})$ and $\mathcal{T}(k, n ; \mathbf{C})$.

Theorem A. Let $A \in \mathrm{Gl}(n ; F)$. The following are equivalent:

I. $A_{k}$ is Morse-Smale.

II. $A_{k}$ is structurally stable.

III. The eigenvalues of $A$ have distinct moduli.

Corollary 1. $A_{k}$ is structurally stable if and only if $A$ is similar to a diagonal matrix whose entries are of increasing modulus.

Proof of Theorem A. It suffices to consider canonical forms.

I $\Rightarrow$ II follows from the theorem of Palis and Smale.

III $\Rightarrow$ I. To show $A_{k}$ is Morse-Smale we must prove:

(1) $\Omega\left(A_{k}\right)$ is finite. 
(2) The periodic points of $A_{k}$ are hyperbolic.

(3) For each $p, q \in \Omega\left(A_{k}\right), W^{u}(p)$ and $W^{s}(q)$ have transversal intersection. Suppose $A=\operatorname{dg}\left(a_{1} \cdots a_{n}\right)$ and $0<\left|a_{i}\right|<\left|a_{i+1}\right|$. Let $p=c_{r_{1}, \ldots, r_{k}}$ be a coordinate plane. Then $p$ is a fixed point of $A_{k}$.

By the lemma

$$
D A_{k}(p)=\operatorname{dg}\left\{\frac{a_{i}}{a_{r_{j}}}\right\} \begin{aligned}
& 1 \leqslant i \leqslant n \\
& 1 \leqslant j \leqslant k
\end{aligned} i \neq r_{l}, \quad l=1, \ldots, k .
$$

Since $\left|a_{i}\right| \neq\left|a_{r_{j}}\right|$ (if $i \neq r_{j}$ ), $p$ is a hyperbolic fixed point of $A_{k}$.

Pick $p \in G(k, n ; F)$ and produce $q=c_{r_{1}}, \ldots, r_{k}$ such that $p \in W^{s}(q)$. If $e$ is a standard chart for $q$, then $p \in \mathcal{C}$. But $A_{k}$ is linear in standard coordinates on the invariant chart $C$. Thus $\Omega\left(A_{k}\right) \cap \mathcal{C}=\{q\}$. So if $p$ is not a coordinate plane, then $p$ wanders.

It remains to prove the transversality condition.

Let $p=c_{\eta}, \ldots, r_{k}$ and $q=c_{s_{1}, \ldots, s_{k}}$ and let $\mathrm{C}$ be the standard chart for $p$, i.e., $\circlearrowright=\left\{\left\{x_{i j}\right\} \mid x_{r_{i} i}=1\right.$ and $x_{r_{i} j}=0$ if $\left.i \neq j\right\}$ where each $\left\{x_{i j}\right\}$ is an $n \times k$ matrix.

$$
W^{u}(p)=\left\{\left\{x_{i j}\right\} \mid\left\{x_{i j}\right\} \in \mathcal{C} \text { and } x_{i j}=0 \text { if } i<r_{j}\right\} \subset \circlearrowright .
$$

Let $\left\{b_{i j}\right\} \in W^{u}(p) \cap W^{s}(q)$ and suppose $\left\{b_{i j}\right\}$ is in $C$ coordinates.

For transversality it suffices to show:

$$
W^{s}(q) \supset\left\{\left\{x_{i j}\right\} \mid\left\{x_{i j}\right\} \in \mathcal{C} \text { and } x_{i j}=b_{i j} \text { if } i \geqslant r_{j}\right\} .
$$

Suppose $\left\{x_{i j}\right\}$ is in the above set. Let $x_{j}$ and $b_{j}$ denote the $j$ th column vectors of $\left\{x_{i j}\right\}$ and $\left\{b_{i j}\right\}$.

Then $\left\{b_{j}\right\}_{j=1}^{k}$ is a basis for $\left\{b_{i j}\right\} \in G(k, n ; F)$. Since $\left\{b_{i j}\right\} \in W^{s}(q)$, there exists another basis $\left\{f_{j}\right\}_{j=1}^{k}$ such that $A^{m}\left(f_{j}\right) \rightarrow c_{s_{j}}$ as $m \rightarrow \infty$.

Each $f_{j}$ can be written as a linear combination,

$$
f_{j}=\sum_{l=1}^{m_{j}} \beta_{l j} b_{l} \quad \text { where } \beta_{m_{j} j} \neq 0 .
$$

Since $\left\{b_{i j}\right\} \in \mathcal{C}, b_{r_{m_{j}} m_{j}}=1$ and $b_{r_{m_{j}} l}=0$ if $l \neq m_{j}$. So the $r_{m_{j}}$ coordinate of the vector $f_{j}$ is

$$
\left(f_{j}\right)_{r_{m}}=\sum_{l=1}^{m_{j}} \beta_{l j} b_{r_{m} l}=\beta_{m_{j} j} \neq 0 .
$$

In general a nonzero vector is forward asymptotic under $A$ to $c_{s_{j}}$ if and only if it is nonzero in the $s_{j}$ place and zero beyond the $s_{j}$ place.

Therefore $s_{j} \geqslant r_{m_{j}}$.

Let $y_{j}=\sum_{l=1}^{m_{j}} \beta_{l j} x_{l}$. Then $\left\{y_{j}\right\}_{j=1}^{k}$ is a basis for $\left\{x_{i j}\right\}$.

The column vectors $x_{l}$ and $b_{l}$ have the same coordinates in the $r_{l}$ place and 
below the $r_{l}$ place. So $f_{j}$ and $y_{j}$ have the same coordinates in the $r_{m_{j}}$ place and below the $r_{m_{j}}$ place. Since $s_{j} \geqslant r_{m_{j}}, f_{j}$ and $y_{j}$ are both forward asymptotic to $c_{s_{j}}$. Therefore $\left\{x_{i j}\right\} \in W^{s}(q)$.

To prove II $\Rightarrow$ III, suppose condition III is not satisfied.

Case 1. $A$ is diagonal and $\left|a_{i}\right|=\left|a_{i+1}\right|$ for some $i$. Let $p=c_{r_{1}, \ldots, r_{k}}$ be a coordinate plane with $r_{j}=i$, but $r_{j+1} \neq i+1$.

By the lemma $D A_{k}(p)$ is a diagonal matrix one of whose entries is $a_{i+1} / a_{i}$. Thus $p$ is a nonhyperbolic fixed point. So $A_{k}$ is not structurally stable.

Case 2. $F=\mathbf{R}$ and $A$ has complex eigenvalues, but all canonical blocks are trivial (i.e., no ones on the superdiagonal).

$A$ can be approximated by a matrix $B$ which is also of the form of Case 2, but whose complex eigenvalues are roots of real numbers. Then for some $m, B^{m}$ is diagonal and at least two of its eigenvalues are of the same modulus.

By Case $1,\left(B_{k}\right)^{m}$ has a nonhyperbolic fixed point. So $B_{k}$ has a nonhyperbolic periodic point. Thus $B_{k}$ is not structurally stable. Since $B_{k}$ approximates $A_{k}, A_{k}$ is not structurally stable.

Case 3. All nontrivial canonical blocks are triangular.

We will approximate $A$ by a matrix whose canonical form is of either Case 1 or 2 .

Suppose $A$ contains the $m$-block ( $a$ may be real or complex)

$$
\left(\begin{array}{cccc}
a & 1 & & 0 \\
& a & 1 & \ddots \\
& 0 & \cdot a & 1 \\
& & & a
\end{array}\right)
$$

Perturb this block to

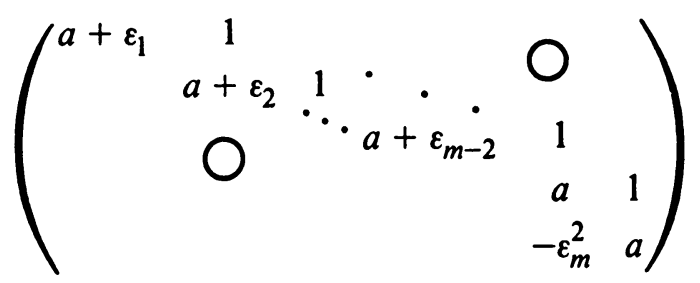

where the $\varepsilon_{i}$ 's are small positive numbers.

If $F=\mathbf{R}$ the canonical form for the perturbation is
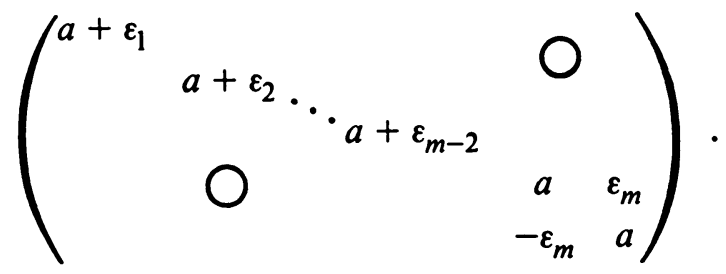
If $F=\mathbf{C}$ the canonical form for the perturbation is (the order may actually be different)

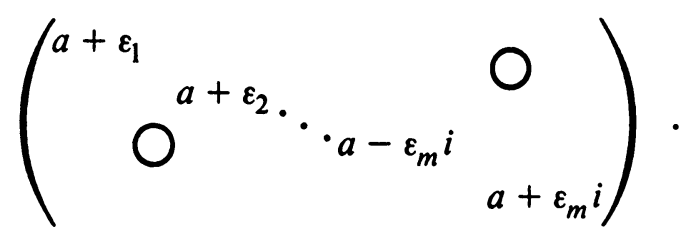

In the above manner, perturb each block of the form

$$
\left(\begin{array}{cccc}
a & 1 & & 0 \\
& \ddots & \ddots & \\
0 & a & 1 \\
& & & a
\end{array}\right)
$$

Let $B$ be the matrix which results from the perturbations and let $C$ be the canonical form for $B$. Then $C$ is of the form of Case 1 or 2 and thus $C_{k}$ is not structurally stable. Since $C_{k}$ is differentiably conjugate to $B_{k}$ and $B_{k}$ approximates $A_{k}, A_{k}$ is not structurally stable.

Case 4. $F=\mathbf{R}$ and $A$ contains nontrivial complex canonical blocks.

Consider the canonical $2 m$-block

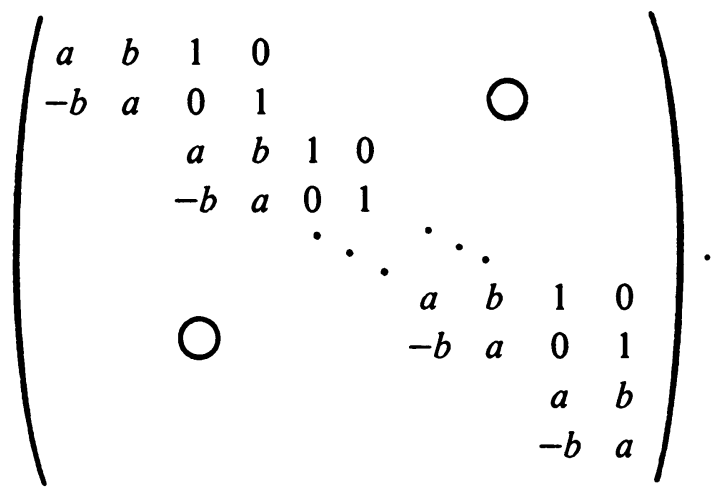

Perturb this block to

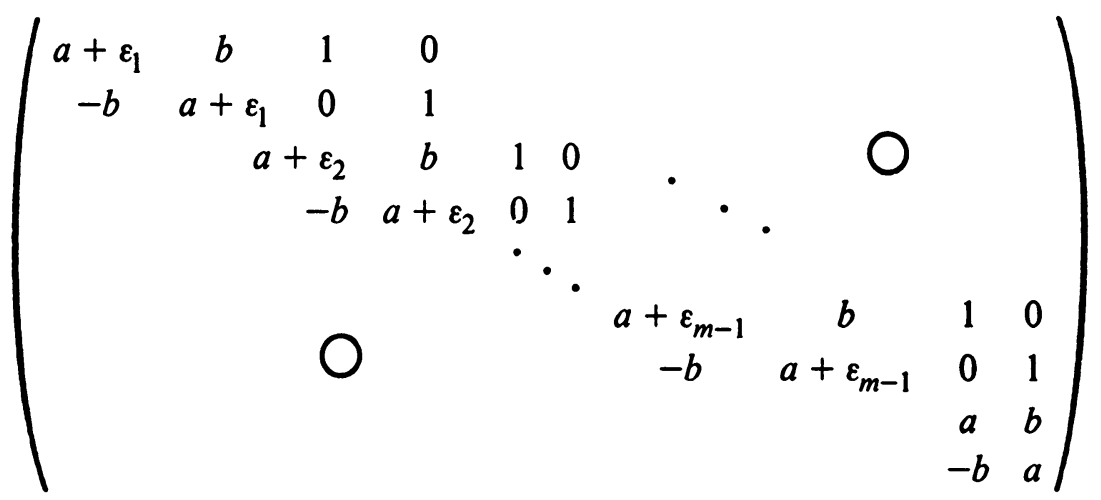


where the $\varepsilon_{i}$ 's are small distinct positive numbers. The canonical form for the perturbation is

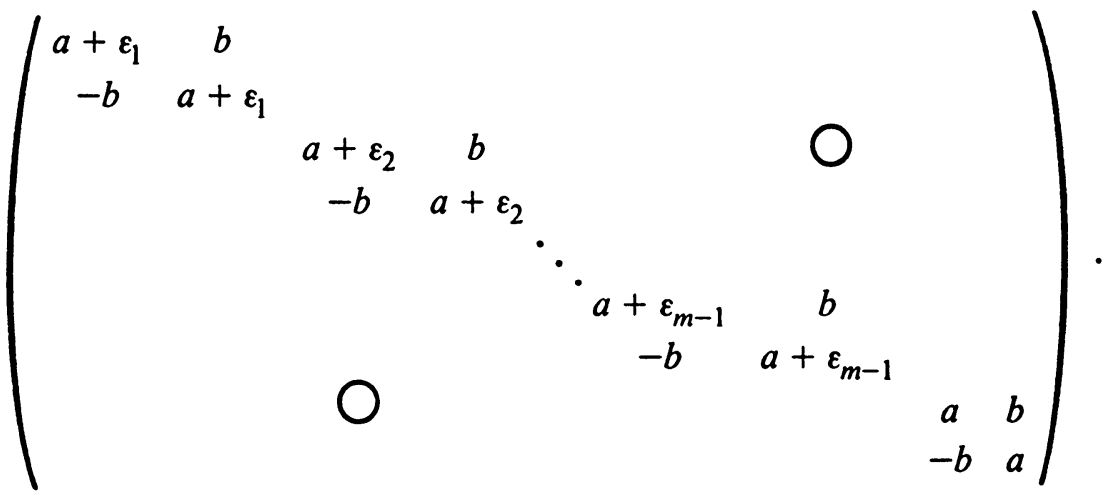

Perturbing each nontrivial complex block in the above manner yields a matrix whose canonical form is that of either Case 2 or Case 3. Therefore $A_{k}$ is not structurally stable. Q.E.D.

Let $\delta(k, n ; F)$ denote the set of structurally stable diffeomorphisms in $\sigma(k, n ; F)$. For the remainder of this section we shall be concerned with the classification of $\delta(k, n ; F)$ up to topological conjugacy.

Lemma. $A_{k}=B_{k}$ if and only if $A=\lambda B$ for some $0 \neq \lambda \in F$.

Proof. If $A=\lambda B$ then clearly $A_{k}=B_{k}$. To prove the "only if" part, we first consider the case where $B=I$.

Suppose $A_{k}=I_{k}$ and $A=\left\{a_{i j}\right\}$.

Let $e_{i}$ be the vector $(0, \ldots, 0,1,0, \ldots, 0)$ where the 1 occurs in the $i$ th place.

Then $A\left(\left\langle e_{1}, \ldots, e_{i-1}, e_{i+1}, \ldots, e_{k+1}\right\rangle\right)=\left\langle e_{1}, \ldots, e_{i-1}, e_{i+1}, \ldots, e_{k+1}\right\rangle$ so

$$
A\left(e_{k+1}\right) \in \bigcap_{i=1}^{k}\left\langle e_{1}, \ldots, e_{i-1}, e_{i+1}, \ldots, e_{k+1}\right\rangle=\left\langle e_{k+1}\right\rangle .
$$

Similarly $A$ maps each $e_{i}$ into $\left\langle e_{i}\right\rangle$. Therefore $A$ is a diagonal matrix. If two of the diagonal entries are not equal, then there is a coordinate plane at which the derivative of $A_{k}$ is not the identity. Thus there exists $0 \neq \lambda \in F$ such that $A=\lambda I$.

Suppose $A_{k}=B_{k}$. Then $\left(A B^{-1}\right)_{k}=A_{k}\left(B_{k}\right)^{-1}=I_{k}$. Produce $0 \neq \lambda \in F$ such that $A B^{-1}=\lambda I$. Then $A=\lambda B$. Q.E.D.

If $Z=\{\lambda I \mid \lambda \in F\}$, then $\mathrm{Gl}(n ; F) / Z$ is a manifold. Define $\Phi: \mathrm{Gl}(n ; F) / Z$ $\rightarrow \sigma(k, n ; F)$ by $\Phi([A])=A_{k}$. The map $\Phi$ is a diffeomorphism (it is injective and well defined by the preceding lemma). Let $S(n ; F)$ be the set of all $A \in \mathrm{Gl}(n ; F)$ such that the eigenvalues of $A$ have distinct moduli. The set $S(n ; F)$ is open in $\mathrm{Gl}(n ; F)$ and thus projects to an open set in $\mathrm{Gl}(n ; F) / Z$. Since $\delta(k, n ; F)$ is the image under $\Phi$ of this projection, $\delta(k, n ; F)$ is an open 
submanifold of $\mathscr{T}(k, n ; F)$. If $F=\mathbf{C}$ then $S(n ; \mathbf{C})$ is dense in $\mathrm{Gl}(n ; \mathbf{C})$ and thus $\delta(k, n ; \mathbf{C})$ is dense in $\mathscr{T}(k, n ; \mathbf{C})$.

Definition. If $A_{k} \in \delta(k, n ; \mathbf{R})$ define $\operatorname{Sgn}(A):\{1, \ldots, n\} \rightarrow\{-1,1\}$ to be the finite sequence given by $\operatorname{Sgn}(A)(i)=\operatorname{sgn}\left(\lambda_{i}\right)$ where $\lambda_{i}$ is the eigenvalue of $A$ of $i$ th largest modulus.

Then $\operatorname{Sgn}(A)$ gives an ordering of the signs of $A$ 's eigenvalues. We now state a corollary to Theorem A.

Corollary 2. If $A_{k}, B_{k} \in \delta(k, n ; \mathbf{R})$ and either $\operatorname{Sgn}(A)=\operatorname{Sgn}(B)$ or $\operatorname{Sgn}(A)$ $=-\operatorname{Sgn}(B)$ then $A_{k} \sim B_{k}$.

Corollary 2 does not give a complete classification of $\delta(k, n ; \mathbf{R})$. Later in this section we will show that if $k>1$, there exist $A_{k}, B_{k} \in \delta(k, 2 k ; \mathbf{R})$ with $A_{k} \sim B_{k}$ but $\operatorname{Sgn}(A) \neq \operatorname{Sgn}(B)$ and $\operatorname{Sgn}(A) \neq-\operatorname{Sgn}(B)$. For structurally stable transformations on complex Grassmann manifolds the situation is simpler.

COROLlaRY 3. $\delta(k, n ; \mathbf{C})$ is an open, dense submanifold of $\sigma(k, n ; \mathbf{C})$ and there is exactly one topological conjugacy class in $\delta(k, n ; \mathbf{C})$.

Proof of Corollary 2. We follow the method of Kuiper [6].

Suppose

$$
\begin{array}{ll}
A=\operatorname{dg}\left(a_{1}, \ldots, a_{n}\right), & \left|a_{j}\right|<\left|a_{j+1}\right|, \\
B=\operatorname{dg}\left(b_{1}, \ldots, b_{n}\right), & \left|b_{j}\right|<\left|b_{j+1}\right|,
\end{array}
$$

and $\operatorname{Sgn}(A)=\operatorname{Sgn}(B)$.

LeMma. If $\left|a_{j-1}\right|<\beta\left|a_{j}\right|$ then

$$
A_{k}=\left(\operatorname{dg}\left(a_{1}, \ldots, a_{n}\right)\right)_{k} \sim\left(\operatorname{dg}\left(a_{1}, \ldots, a_{j-1}, \beta a_{j}, \beta a_{j+1}, \ldots, \beta a_{n}\right)\right)_{k} .
$$

Before proving the lemma we will complete the proof of the corollary.

Produce $\beta_{1}, \ldots, \beta_{n}>0$ such that

$$
\begin{aligned}
b_{1} & =\beta_{1} a_{1}, \\
b_{2} & =\beta_{1} \beta_{2} a_{2}, \\
& \vdots \\
b_{n} & =\beta_{1} \beta_{2} \cdots \beta_{n} a_{n} ;
\end{aligned}
$$

then applying the lemma: 


$$
\begin{aligned}
A_{k}= & \left(\operatorname{dg}\left(a_{1}, \ldots, a_{n}\right)\right)_{k} \\
= & \left(\operatorname{dg}\left(\beta_{1} a_{1}, \ldots, \beta_{1} a_{n}\right)\right)_{k} \\
= & \left(\operatorname{dg}\left(b_{1}, \beta_{1} \alpha_{2}, \ldots, \beta_{1} a_{n}\right)\right)_{k} \\
\sim & \left(\operatorname{dg}\left(b_{1}, \beta_{1} \beta_{2} a_{2}, \ldots, \beta_{1} \beta_{2} a_{n}\right)\right)_{k} \\
& \quad \text { since }\left|b_{1}\right|<\left|b_{2}\right|=\left|\beta_{1} \beta_{2} a_{2}\right|=\beta_{2}\left|\beta_{1} a_{2}\right| \\
= & \left(\operatorname{dg}\left(b_{1}, b_{2}, \ldots, \beta_{1} \beta_{2} a_{n}\right)\right)_{k} \\
\vdots & \\
\sim & \left(\operatorname{dg}\left(b_{1}, b_{2}, \ldots, b_{n-1}, \beta_{1} \beta_{2} \ldots \beta_{n} a_{n}\right)\right)_{k} \\
= & \left(\operatorname{dg}\left(b_{1}, \ldots, b_{n}\right)\right)_{k} \\
= & B_{k} .
\end{aligned}
$$

If $\operatorname{Sgn}(A)=-\operatorname{Sgn}(B)$, then $A_{k} \sim(-B)_{k}=B_{k}$.

It remains to prove the lemma.

Suppose $\left|a_{j-1}\right|<\beta\left|a_{j}\right|$. For each real number $t$ between 1 and $\beta$, define $A^{t}=\operatorname{dg}\left(a_{1}, \ldots, a_{j-1}, t a_{j}, t a_{j+1}, \ldots, t a_{n}\right)$.

Note. $A^{1}=A$ and $\left(A^{l}\right)_{k} \in \mathcal{S}(k, n ; \mathbf{R})$.

Thus $\left\{\left(A^{t}\right)_{k}\right\}$ is a path of structurally stable diffeomorphisms between the two diffeomorphisms which we want to show are conjugate. Since structural stability is an open condition, the two diffeomorphisms are topologically conjugate.

In proving Corollary 2 we have actually shown that if $\operatorname{Sgn}(A)=\operatorname{Sgn}(B)$ then $A_{k}$ and $B_{k}$ are in the same path component of $\delta(k, n ; \mathbf{R})$. The proof of the second part of Corollary 3 is similar and amounts to showing that $\delta(k, n ; \mathbf{C})$ is path connected. The hypothesis of the lemma is changed to $\left|a_{j-1}\right|<\left|\beta a_{j}\right|$ where $\beta$ is a complex number. To prove the new lemma, define $A^{t}$ by choosing a path of $t$ 's in $\mathbf{C}$ between one and $\beta$ such that the modulus of each point on the path is between 1 and $|\beta|$.

For the remainder of this section we shall be concerned with the topological classification of $\delta(k, n ; \mathbf{R})$. We begin by showing the relationship between $\delta(k, n ; \mathbf{R})$ and $\delta(n-k, n ; \mathbf{R})$. Recall that if $A_{k} \in \delta(k, n ; \mathbf{R})$ then $\operatorname{Sgn}(A)$ : $\{1, \ldots, n\} \rightarrow\{-1,1\}$ gives the ordering of the signs of the eigenvalues (from the smallest to largest). Note that $\operatorname{Sgn}\left(A^{-1}\right)$ merely reverses the ordering of $\operatorname{Sgn}(A)$.

THEOREM B. If $A_{k} \in \delta(k, n ; \mathbf{R})$ and $B_{n-k} \in \delta(n-k, n ; \mathbf{R})$ and either $\operatorname{Sgn}\left(A^{-1}\right)=\operatorname{Sgn}(B)$ or $\operatorname{Sgn}\left(A^{-1}\right)=-\operatorname{Sgn}(B)$ then $A_{k} \sim B_{n-k}$.

EXAMPLES. 


$$
\begin{aligned}
& (\operatorname{dg}(12334-5))_{2} \sim(\operatorname{dg}(-123345))_{3} \text {, } \\
& (\operatorname{dg}(1-234))_{2} \sim\left(\operatorname{dg}\left(\begin{array}{llll}
1 & 2 & -3 & 4
\end{array}\right)\right)_{2} \text {. }
\end{aligned}
$$

Proof. We can assume $A=\operatorname{dg}\left(a_{1} \cdots a_{n}\right),\left|a_{i}\right|<\left|a_{i+1}\right|$. Let $d_{k}: G(k, n ; \mathbf{R})$ $\rightarrow G(n-k, n ; \mathbf{R})$ be the diffeomorphism that maps a $k$-dimensional subspace of $\mathbf{R}^{n}$ to its $(n-k)$-dimensional orthogonal complement.

Lemma. $d_{k}$ is a conjugacy for $A_{k}$ and $\left(A^{-1}\right)_{n-k}$.

Proof of lemma. $A^{-1}=\operatorname{dg}\left(a_{1}^{-1} \cdots a_{n}^{-1}\right)$.

Let $p \in G(k, n ; \mathbf{R})$ and suppose $\vec{u}$ is a vector in $p$. Suppose $\vec{r}$ is a vector in $d_{k}(p) \in G(n-k, n ; \mathbf{R})$. If $\vec{u}=\left(u_{1}, \ldots, u_{n}\right)$ and $\vec{r}=\left(r_{1}, \ldots, r_{n}\right)$ then $A(\vec{u})=\left(a_{1} u_{1}, \ldots, a_{n} u_{n}\right)$ and $A^{-1}(\vec{r})=\left(a_{1}^{-1} r_{1}, \ldots, a_{n}^{-1} r_{n}\right)$, and $\vec{u} \cdot \vec{r}=$ 0 .

$$
A(\vec{u}) \cdot A^{-1}(\vec{r})=\left(u_{1} r_{1}, \ldots, u_{n} r_{n}\right)=\vec{u} \cdot \vec{r}=0 .
$$

So $A$ applied to a vector $\vec{u}$ in $p$ is orthogonal to $A^{-1}$ applied to a vector which is orthogonal to $\vec{u}$. But the choice of vectors was arbitrary. Therefore the diagram commutes:

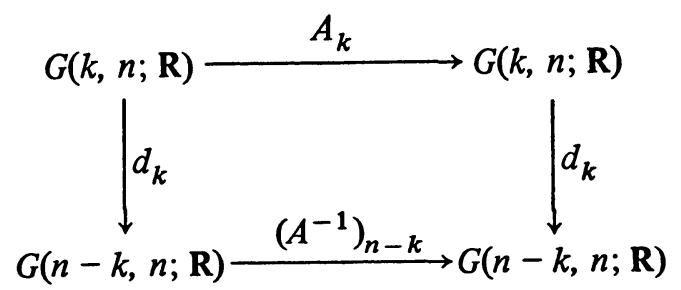

Returning to the theorem, suppose $\operatorname{Sgn}\left(A^{-1}\right)=\operatorname{Sgn}(B)$. Then by Corollary 2, $\left(A^{-1}\right)_{n-k} \sim B_{n-k}$. So by the lemma, $A_{k} \sim B_{n-k}$. If $\operatorname{Sgn}\left(A^{-1}\right)=-\operatorname{Sgn}(B)$, then $A_{k} \sim(-B)_{n-k}=B_{n-k}$.

For $n=2 k$ Theorem B gives some conditions sufficient for the topological equivalence of elements in $\delta(k, n ; \mathbf{R})$. We shall now show that these conditions combined with those given in Corollary 2 are necessary. This will complete the classification of $\delta(k, n ; \mathbf{R})$.

For the remainder of this chapter assume unless stated otherwise that $A_{k}, B_{k} \in \delta(k, n ; \mathbf{R})$ and that $A$ and $B$ are in canonical form. Since $A_{k}$ and $B_{k}$ are also Morse-Smale we can define their diagrams.

If $p, q \in \operatorname{Fix}\left(A_{k}\right)$ then $p \leqslant q$ if and only if $W^{u}(p) \cap W^{s}(q) \neq \varnothing$. If $p \leqslant q$ and $p \neq q$, then $p<q$. A diagram of $A_{k}$ consists of vertices corresponding to fixed points and oriented segments from vertex $p$ to $q$ (denoted $p \rightarrow q$ ) when $p<q$, but there is no $q^{\prime}$ such that $p<q^{\prime}<q$. If $p \rightarrow q$ then $p$ is said to give to $q$ and $q$ is said to receive from $p$.

The following lemma describes the diagram structure of a canonical form 
structurally stable transformation. Lemma a is also valid for $F=\mathbf{C}$.

LEMMA a. (1) $c_{r_{1}, \ldots, r_{k}} \leqslant c_{s_{1}, \ldots, s_{k}}$ if and only if, for each $i, r_{i} \leqslant s_{i}$.

(2) $c_{\eta}, \ldots, \eta_{k} \rightarrow c_{s_{1}}, \ldots, s_{k}$ if and only if there exists $1 \leqslant j \leqslant k$ such that $r_{j}=s_{j}-1$ and $r_{i}=s_{i}$ for $i \neq j$.

(3) $A$ vertex can give to at most $k$ other vertices and can receive from at most $k$ other vertices.

Proof. For (1) look at $W^{u}\left(c_{r_{1}, \ldots, r_{k}}\right) \subset \mathcal{C}$ where $\mathcal{C}$ is the standard chart for $c_{r_{1}}, \ldots, \eta_{k}$. Consider what happens to a point in $W^{u}\left(c_{r_{1}}, \ldots, \eta_{k}\right)$ under forward iteration.

(3) follows from (2) which follows from (1).

Lemma b. If $p \rightarrow q$, then $\left[W^{u}(p) \cap W^{s}(q)\right] \cup\{p, q\}$ is a circle which is invariant under $A_{k}$. Also $p$ and $q$ are the only fixed points of $A_{k} \mid\left(\left[W^{u}(p)\right.\right.$ $\left.\left.\cap W^{s}(q)\right] \cup\{p, q\}\right)$.

The diagrams that we have defined are special cases of the Morse-Smale diagrams which Smale describes in his survey article [15]. Our labelling of the diagrams will be different from that of Smale.

Label $p \rightarrow q$ with a "+" ("-") if $A_{k}$ preserves (reverses) orientation on $\left(\left[W^{u}(p) \cap W^{s}(q)\right] \cap\{p, q\}\right)$. Lemma a implies that without labelling, the diagram is completely determined by $n$ and $k$. The diagrams of $A_{k}$ and $B_{k}$ will be defined to be equal provided that they have the same labelling.

LEMma c. (1) The labelling of $c_{r_{1}}, \ldots, r_{k} \rightarrow c_{r_{1}}, \ldots, r_{i-1}, r_{i}+1, \ldots, r_{k}$ is the sign of $a_{r_{i}} a_{r_{i}+1}$.

(2) $A_{k}$ and $B_{k}$ have equal diagrams if and only if either $\operatorname{Sgn}(A)=\operatorname{Sgn}(B)$ or $\operatorname{Sgn}(A)=-\operatorname{Sgn}(B)$.

Definition. The jth row in the diagram of $A_{k}$ is the set of fixed points with stable manifolds of dimension $j$.

LEMMA d. The fixed point $c_{\eta, \ldots, r_{k}}$ is in row $\left(\sum_{i=1}^{k} r_{i}-k(k+1) / 2\right)$.

DEFINITION. $H$ : $\operatorname{Fix}\left(A_{k}\right) \rightarrow \operatorname{Fix}\left(B_{k}\right)$ is a diagram isomorphism if and only if:

(a) $\operatorname{dim}\left(W^{s}(H(p))\right)=\operatorname{dim}\left(W^{s}(p)\right)$ for any $p \in \operatorname{Fix}\left(A_{k}\right)$.

(b) $p \rightarrow q \Leftrightarrow H(p) \rightarrow H(q)$.

(c) If $p \rightarrow q$ then $\operatorname{sgn}\{p, q\}=\operatorname{sgn}\{H(p), H(q)\}$ where $\operatorname{sgn}\{p, q\}$ is the sign $(+$ or -$)$ of the diagram arrow.

For the definition of diagram isomorphism we will not require $A$ and $B$ to be in canonical form. If $A$ and $B$ are in canonical form then $\operatorname{Fix}\left(A_{k}\right)$ $=\operatorname{Fix}\left(B_{k}\right)$. In this case we shall say that the diagram isomorphism is a diagram automorphism on the diagram of $A_{k}$.

\section{EXAMPLES.}


(1) $\left(\operatorname{dg}\left(\begin{array}{lll}-1 & 2 & 3\end{array}\right)\right)_{2}$

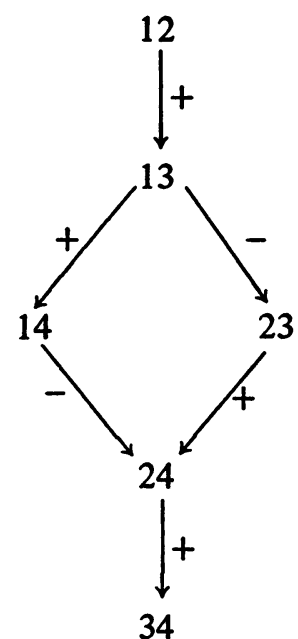

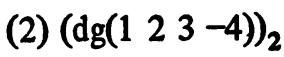

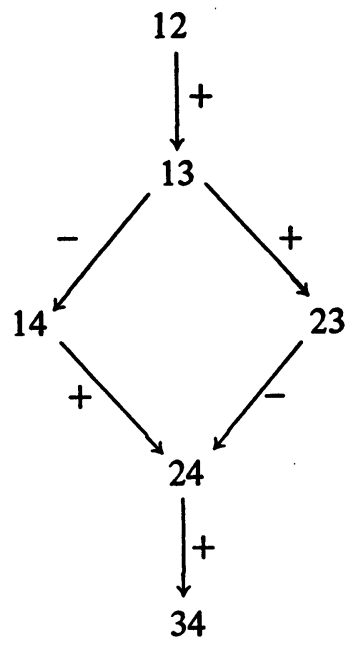

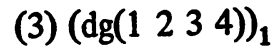

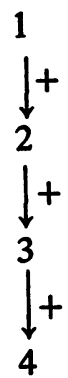

The diagrams of examples (1) and (2) are isomorphic. The diagram isomorphism is also a diagram automorphism.

(4) $(\operatorname{dg}(-122345))_{2} \in \S(2,5 ; R)$

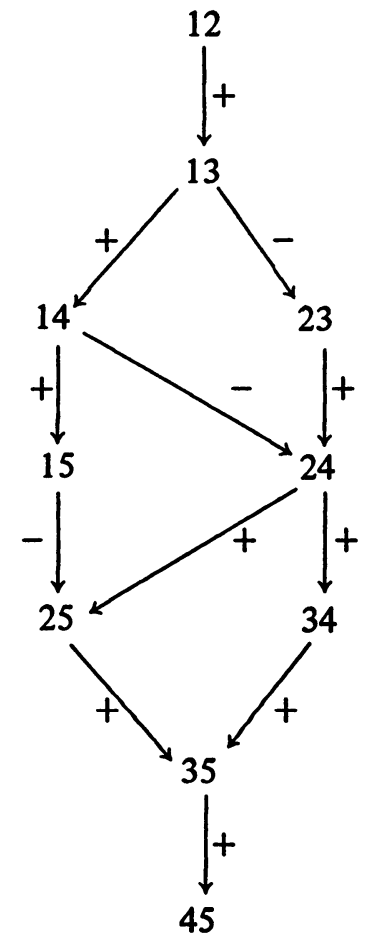


Note. The only diagram automorphism on the diagram of example (4) is the identity.

We shall use the diagrams to distinguish maps which are not topologically equivalent. We now give an example of two diffeomorphisms in $\delta(2,4 ; \mathbf{R})$ whose diagrams are not isomorphic. Using Smale's definition of labelling, these same maps have diagrams that are equal. The diffeomorphisms are not conjugate.

$$
\left(\operatorname{dg}\left(\begin{array}{llll}
1 & 2 & 3 & 4
\end{array}\right)\right)_{2}
$$

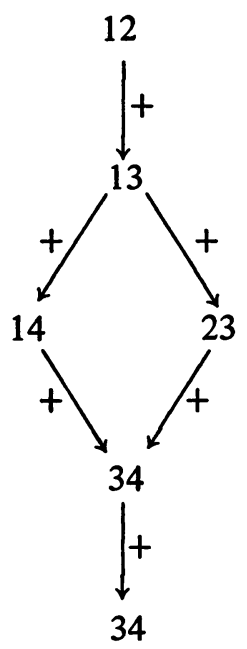

$$
(\operatorname{dg}(123-4))_{2}
$$

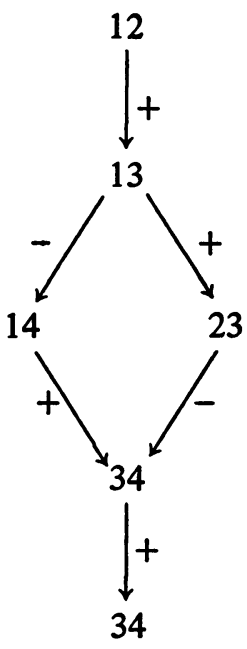

Same maps with Smale diagrams

$\operatorname{dim} W^{s} \operatorname{dim} W^{u}$

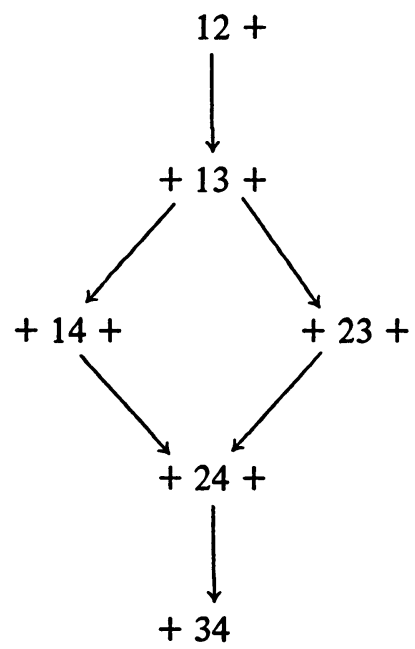

0

4

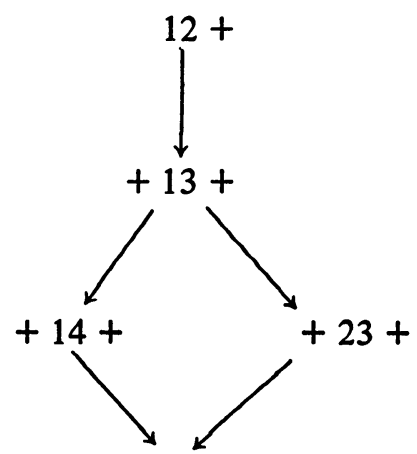

3

$+24+$

4

0

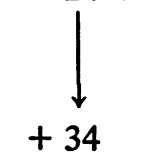


The sign on the left (right) side of the vertex indicates whether the map preserves or reverses orientation on the stable (unstable) manifold of the fixed point.

If $k \geqslant 2$ the first three rows (without labelling) in the diagram of $A_{k}$ are:

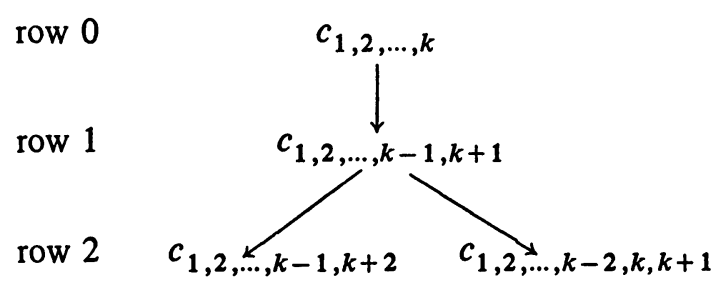

Lemma e. If $H$ is a diagram automorphism on the diagram of $A_{k}$, then $H$ is determined by its restriction to row 2 (i.e., if $A, B, C$ are in canonical form and $A_{k}, B_{k}, C_{k} \in \delta(k, n ; \mathbf{R})$ and $H: \operatorname{Fix}\left(A_{k}\right) \rightarrow \operatorname{Fix}\left(B_{k}\right), H^{\prime}: \operatorname{Fix}\left(A_{k}\right) \rightarrow \operatorname{Fix}\left(C_{k}\right)$ are diagram automorphisms whose restrictions to row 2 are equal, then the diagrams of $B_{k}$ and $C_{k}$ are equal).

Proof. If $k=1$, each row has only one element. Thus for $k=1$, the identity is the only diagram automorphism.

Suppose $k>1$ and $H$ is a diagram automorphism. Then row 0 and row 1 each have only one element which therefore must be fixed by $H$.

To prove the lemma, it suffices to show that in each row $j(j \geqslant 3)$ there are no two elements which receive from exactly the same vertices.

Suppose $p$ and $p^{\prime}$ are in row $j \geqslant 3$ and that both $p$ and $p^{\prime}$ receive from $q$ in row $j-1$.

Let $p=c_{\eta}, \ldots, r_{k}$. Then $q$ is of the form $q=c_{r_{1}, \ldots, r_{m-1}, r_{m}-1, r_{m+1}, \ldots, r_{k}}$ and $p^{\prime}$ is of the form $p^{\prime}=c_{r_{1}}, \ldots, \eta_{-1}, \eta_{1}+1, \eta_{+1}, \ldots, r_{m-1}, r_{m}-1, r_{m+1}, \ldots, r_{k}$.

So the indices of $p$ and $p^{\prime}$ agree at all spots except for $l$ and $m$ where they differ by 1 . It would be impossible to find a $q^{\prime} \neq q$ that gives to both $p$ and $p^{\prime}$.

It remains to show that there cannot exist $p$ and $p^{\prime}$ in row $j(j \geqslant 3)$ which both receive from $q$ and only $q$.

If $p$ receives from exactly one vertex, it has one of the following two forms:

(i) $p=c_{r_{1}, r_{1}+1, \ldots, r_{1}+k-1}$;

(ii) $p=c_{1, \ldots, m-1, r_{m}, r_{m}+1, \ldots, r_{m}+k-m}, r_{m}>m$.

Clearly there do not exist $p$ and $p^{\prime}$ in row $j$ such that both $p$ and $p^{\prime}$ are of form (i).

Suppose

$$
p=c_{1, \ldots, m-1, r_{m}, r_{m}+1, \ldots, r_{m}+k-m}, \quad r_{m}>m
$$

Then

$$
q=c_{1, \ldots, m-1, r_{m}-1, r_{m}+1, \ldots, r_{m}+k-m} .
$$


Now $p^{\prime}$ can only have one gap in its sequence and $p^{\prime}$ must be derived from raising by one an element in the sequence for $q$. Since there is a gap between $r_{m}-1$ and $r_{m}+1$ in the sequence for $q$, that sequence must stop at $r_{m}-1$ or else there would be two gaps in the sequence for $p^{\prime}$. This forces $m=k$.

Thus $p=c_{1, \ldots, k-1, r_{k}}, q=c_{1, \ldots, k-1, r_{k}-1}$. Then $p^{\prime}=c_{1, \ldots, k-2, k, r_{k}-1}$.

Since $p^{\prime}$ can only have one gap in its sequence, $r_{k}-1=k+1$. This means $p^{\prime}=c_{1, \ldots, k-2, k, k+1}$. But then $p^{\prime}$ is in row 2. Q.E.D.

COROLLARY. There are either 1 or 2 automorphisms on the diagram of $A_{k}$.

Proof. The identity is always a diagram automorphism. There are only 1 or 2 elements in row 2 , so there can be at most 2 automorphisms.

We are now ready to classify $\delta(k, n ; \mathbf{R})$.

Theorem C. Let $A_{k}, B_{k} \in \delta(k, n ; \mathbf{R})$. The following are equivalent:

I. $A_{k} \sim B_{k}$.

II. diagram $\left(A_{k}\right)$ is isomorphic to diagram $\left(B_{k}\right)$.

III. One of the following is true:

(i) $\operatorname{Sgn}(A)=\operatorname{Sgn}(B)$,

(ii) $\operatorname{Sgn}(A)=-\operatorname{Sgn}(B)$,

(iii) $n=2 k$ and $\operatorname{Sgn}\left(A^{-1}\right)=\operatorname{Sgn}(B)$,

(iv) $n=2 k$ and $\operatorname{Sgn}\left(A^{-1}\right)=-\operatorname{Sgn}(B)$.

The theorem does not require that $A$ and $B$ be in canonical form.

PRoof. III $\Rightarrow$ I follows from Corollary 2 and Theorem B.

To prove I $\Rightarrow$ II we must show that diagram isomorphism is a topological invariant.

Let $h$ be a conjugacy for $A_{k}$ and $B_{k}$ and let $H: \operatorname{Fix}\left(A_{k}\right) \rightarrow \operatorname{Fix}\left(B_{k}\right)$ be defined by $H=h \mid$ Fix $\left(A_{k}\right)$.

Claim. $H$ is a diagram isomorphism.

(a) $W^{s}(H(p))=h\left(W^{s}(p)\right)$. So $\operatorname{dim}\left(W^{s}(H(p))\right)=\operatorname{dim}\left(W^{s}(p)\right)$.

(b) Suppose $p \leqslant q$ (that is, $\left.W^{u}(p) \cap W^{s}(q) \neq \varnothing\right)$.

$$
\begin{aligned}
W^{u}(H(p)) \cap W^{s}(H(q)) & =h\left(W^{u}(p)\right) \cap h\left(W^{s}(q)\right) \\
& =h\left(W^{u}(p) \cap W^{s}(q)\right) \\
& \neq \varnothing .
\end{aligned}
$$

Thus $H(p) \leqslant H(q)$ and $H$ preserves the partial ordering (as does $H^{-1}$ ). Therefore condition (b) is satisfied.

(c) Suppose $p \rightarrow q$. Then $h \mid\left(\{p, q\} \cup\left[W^{u}(p) \cap W^{s}(q)\right]\right)$ is a conjugacy. So $H$ must preserve $\operatorname{sgn}\{p, q\}$.

II $\Rightarrow$ III. Assume $A_{k}$ and $B_{k}$ are in canonical form.

Case 1. Suppose $n>2 k$ and neither $\operatorname{Sgn}(A)=\operatorname{Sgn}(B)$ nor $\operatorname{Sgn}(A)$ 
$=-\operatorname{Sgn}(B)$. To show diagram $\left(A_{k}\right)$ is not isomorphic to diagram $\left(B_{k}\right)$, it suffices to show that the identity is the only diagram automorphism on diagram $\left(A_{k}\right)$.

Let $H$ be a diagram automorphism on the diagram of $A_{k}$.

Claim. The restriction of $H$ to the second row in the diagram of $A_{k}$ is the identity.

Consider $c_{2,3, \ldots, k, k+1}$ in row $k$. This vertex receives from exactly 1 and gives to exactly 1 vertex. Because $n>2 k$, it is the only vertex in row $k$ which has those properties. Consequently $c_{2,3, \ldots, k_{1} k+1}$ must be fixed under $H$.

Define a finite sequence $\left\{s_{i}\right\}_{i=1}^{k}$ from $s_{1}=c_{2,3, \ldots, k, k+1}$ to $s_{k-1}$ $=c_{1, \ldots, k-2, k, k+1}$ by letting $s_{i+1}$ be the only vertex that gives to $s_{i}$ (i.e., $\left.s_{i}=c_{1,2, \ldots, i-1, i+1, \ldots, k, k+1}\right)$.

Since $s_{1}$ is fixed under $H$, each element in the sequence is also fixed under $H$. In particular $s_{k-1}=c_{1, \ldots, k-2, k, k+1}$ is a fixed point. But $s_{k-1}$ is one of the two elements in row 2. So $H$ restricted to the second row in the diagram of $A_{k}$ is the identity.

By Lemma e, $H$ is the identity.

Case 2. $k<n<2 k$ and neither $\operatorname{Sgn}(A)=\operatorname{Sgn}(B)$ nor $\operatorname{Sgn}(A)$ $=-\operatorname{Sgn}(B)$.

Let $k^{\prime}=n-k$. Then $n>2 k^{\prime}$. Let $A^{-}=\operatorname{dg}\left(a_{n}^{-1}, \ldots, a_{1}^{-1}\right)$ and $B^{-}$ $=\operatorname{dg}\left(b_{n}^{-1}, \ldots, b_{1}^{-1}\right)$.

Then $A^{-}$and $B^{-}$are in canonical form and neither $\operatorname{Sgn}\left(A^{-}\right)=\operatorname{Sgn}\left(B^{-}\right)$ nor $\operatorname{Sgn}\left(A^{-}\right)=-\operatorname{Sgn}\left(B^{-}\right)$. By Theorem B, $A_{k} \sim\left(A^{-}\right)_{k^{\prime}}$ and $B_{k} \sim\left(B^{-}\right)_{k^{\prime}}$.

But from Case 1, diagram $\left(A^{-}\right)_{k^{\prime}}$ is not isomorphic to diagram $\left(B^{-}\right)_{k^{\prime}}$.

By a slight generalization of "diagram isomorphism" and an argument similar to $\mathrm{I} \Rightarrow \mathrm{II}$, diagram $\left(A^{-}\right)_{k^{\prime}}$ is isomorphic to diagram $\left(A_{k}\right)$ and diagram $\left(B^{-}\right)_{k^{\prime}}$ is isomorphic to diagram $\left(B_{k}\right)$.

Therefore diagram $\left(A_{k}\right)$ is not isomorphic to diagram $\left(B_{k}\right)$.

Case 3. $n=2 k$.

Let $A=\operatorname{dg}\left(a_{1}, \ldots, a_{n}\right), A^{-1}=\operatorname{dg}\left(a_{1}^{-1}, \ldots, a_{n}^{-1}\right), A^{-}=\operatorname{dg}\left(a_{n}^{-1}, \ldots, a_{1}^{-1}\right)$. We will show that the conjugacy between $A_{k}$ and $\left(A^{-}\right)_{k}$ induces a nonidentity diagram automorphism.

In the conjugacy $d_{k}$ from $A_{k}$ to $\left(A^{-1}\right)_{k}, d_{k}$ was defined by taking orthogonal complements. Thus $d_{k}\left(c_{1, \ldots, k-1, k+2}\right)=c_{k, k+1, k+3, k+4, \ldots, 2 k}$.

Let $P=\left\{P_{i j}\right\}$ be the $n \times n$ matrix defined by $P_{i j}=1$ if $i+j=n+1$ and $P_{i j}=0$ otherwise. $P_{k}$ is a differentiable conjugacy for $\left(A^{-1}\right)_{k}$ and $\left(A^{-}\right)_{k}$.

$$
P_{k}\left(c_{k, k+1, k+3, k+4, \ldots, 2 k}\right)=c_{1, \ldots, k-2, k, k+1} .
$$

Thus there is a conjugacy between $A_{k}$ and $\left(A^{-}\right)_{k}$ whose restriction $H$ to the fixed point set has the property that $H\left(c_{1, \ldots, k-1, k+2}\right)=c_{1, \ldots, k-2, k, k+1}$. But $H$ is a diagram automorphism. So we have a nonidentity diagram automor- 
phism produced by the conjugacy from $A_{k}$ to $\left(A^{-}\right)_{k}$.

Since $\operatorname{Sgn}\left(A^{-1}\right)=\operatorname{Sgn}\left(A^{-}\right)$, conditions (iii) and (iv) generate the only possible nonidentity automorphism.

Since $\operatorname{Sgn}(A)$ is invariant up to similarity of the matrix $A$, II $\Rightarrow$ III is true regardless of whether $A_{k}$ and $B_{k}$ are in canonical form.

\section{BIBLIOGRAPHY}

1. C. G. Cullen, Matrices and linear transformations, Addison-Wesley, Reading, Mass., 1966. MR 33 \#5637.

2. C. Ehresmann, Sur la topologie de certains espaces homogènes, Ann. of Math. (2) 35 (1934), 396-443.

3. J. Franks, Necessary conditions for stability of diffeomorphisms, Trans. Amer. Math. Soc. 158 (1971), 301-308. MR 44 \#1042.

4.

5. M. W. Hirsch, C. C. Pugh and M. Shub, Invariant manifolds, Bull. Amer. Math. Soc. 76 (1970), 1015-1019. MR 45 \# 1188.

6. N. Kuiper, Topological conjugacy of real projective transformations, Inst. Hautes Études Sci. Publ. Math., 1974 (preprint).

7. N. H. Kuiper and J. W. Robbin, Topological classification of linear endomorphisms, Invent. Math. 19 (1973), 83-106. MR 47 \#8567.

8. J. Milnor and J. Stasheff, Characteristic classes, Ann. of Math. Studies, no. 76, Princeton Univ. Press, Princeton, N.J., 1974.

9. Z. Nitecki, Differentiable dynamics, MIT Press, Cambridge, Mass., 1971.

10. J. Palis and S. Smale, Structural stability theorems, Proc. Sympos. Pure Math., vol. 14, Amer. Math. Soc., Providence, R.I., 1970, 223-231. MR 42 \#2505.

11. J. W. Robbin, A structural stability theorem, Ann. of Math. (2) 94 (1971), 447-493. MR 44 \#4783.

12. - Topological conjugacy and structural stability for discrete dynamical systems, Bull. Amer. Math. Soc. 78 (1972), 923-952. MR 47 \#1086.

13. R. C. Robinson, Structural stability of $C^{1}$ diffeomorphisms, J. Differential Equations 22 (1976), 28-73.

14. C. Simon, Non-genericity of rational zeta functions and instability in $\operatorname{Diff}^{r}\left(T^{3}\right)$, Thesis, Northwestern Univ., 1970.

15. S. Smale, Differentiable dynamical systems, Bull. Amer. Math. Soc. 73 (1967), 747-817. MR 37 \#3598; erratum, 39, p. 1593.

16. Stable manifolds for differential equations and diffeomorphisms, Ann. Scuola Norm. Sup. Pisa (3) 17 (1963), 97-116. MR 29 \#2818b.

17. F. W. Warner, Foundations of differentiable manifolds and Lie groups, Scott-Foresman, Glenview, Ill., 1971. MR 45 \#4312.

Department of Mathematics, Northwestern University, Evanston, Illinois 60201

Current address: Department of Mathematics, Emory University, Atlanta, Georgia 30322 